\title{
Compressible phase of a double-layer electron system with total Landau-level filling factor one-half
}

\author{
N. E. Bonesteel \\ National High Magnetic Field Laboratory, Florida State University, P.O. Box 4005, Tallahassee, \\ FL 32306-4005
}

\begin{abstract}
Following recent work of Halperin, Lee, and Read, and Kalmeyer and Zhang, a double-layer electron system with total Landau-level filling factor $\nu=1 / 2$ is mapped onto an equivalent system of fermions in zero average magnetic field interacting via a Chern-Simons gauge field. Within the random-phase approximation a new, low-lying, diffusive mode, not present in the $\nu=1 / 2$ single-layer system, is found. This mode leads to more singular low-energy scattering than appears in the single layer system, and to an attractive pairing interaction between fermions in different layers which grows stronger as the layer spacing is decreased. The possible connection between this pairing interaction and the experimentally observed fractional quantum Hall effect in double-layer systems is discussed.
\end{abstract}

Typeset using REVTEX 
Recently, the fractional quantum Hall effect (FQHE) has been seen in double-layer electron systems with total Landau-level filling factor $\nu=1 / 2$ [1,2]. This observation supports the long-held belief that incompressible, even-denominator quantum Hall states can exist when there are two species of fermions [3 6]. However, in these systems, as the ratio of the layer spacing, $d$, to the magnetic length, $l_{0}\left(\equiv\left(\hbar c / e B_{0}\right)^{1 / 2}\right.$, where $B_{0}$ is the applied magnetic field), is increased, the FQHE becomes weaker and eventually disappears [1], indicating that the state has become compressible. The $\nu=1 / 2$ single-layer system, which is also compressible [7], has been described by Halperin, Lee, and Read [8], and Kalmeyer and Zhang [9], in terms of a 'Fermi liquid' of electrons bound to an even number of flux quanta. It is the purpose of this paper to develop a similar description for the compressible phase of the $\nu=1 / 2$ double-layer system, and to point out some new features of this description which are not present in the single layer case.

Specifically, we consider a double-layer system in a transverse magnetic field, $B_{0}=$ $4 h c n / e$, where $n$ is the carrier density per layer, (for the remainder of this paper $\hbar=c=1$, so that, e.g., $\left.B_{0}=8 \pi n / e\right)$. The total Landau-level filling factor is then $\nu=1 / 2$, and the filling factor in each layer is $\nu=1 / 4$. Electron spins are assumed to be fully polarized, and tunneling between layers is ignored.

In two dimensions, it is possible to continuously change the statistics of identical particles by attaching infinitely thin flux tubes containing fictitious flux to them [10]. In this paper we will refer to this fictitious flux as Chern-Simons flux, and to the transformed particles as pseudo-particles. In double-layer systems it is useful to introduce two types of ChernSimons flux. The relationship between physical electrons and pseudo-particles can then be expressed mathematically as a 'singular gauge transformation' of the form

$$
\begin{aligned}
& \psi_{1}(\mathbf{r})=\psi_{e, 1}(\mathbf{r}) \exp \left(i \phi_{1} \int d^{2} r^{\prime} \arg \left(\mathbf{r}-\mathbf{r}^{\prime}\right) \rho_{1}\left(\mathbf{r}^{\prime}\right)+i \phi_{2} \int d^{2} r^{\prime} \arg \left(\mathbf{r}-\mathbf{r}^{\prime}\right) \rho_{2}\left(\mathbf{r}^{\prime}\right)\right) \\
& \psi_{2}(\mathbf{r})=\psi_{e, 2}(\mathbf{r}) \exp \left(i \phi_{2} \int d^{2} r^{\prime} \arg \left(\mathbf{r}-\mathbf{r}^{\prime}\right) \rho_{1}\left(\mathbf{r}^{\prime}\right)+i \phi_{1} \int d^{2} r^{\prime} \arg \left(\mathbf{r}-\mathbf{r}^{\prime}\right) \rho_{2}\left(\mathbf{r}^{\prime}\right)\right)
\end{aligned}
$$

where $\psi_{e, s}(r)$ and $\psi_{s}(r)$ are, respectively, the physical electron and pseudo-particle annihilation operators in layer $s, \rho_{s}(r)$ is the density operator in layer $s$, and $\arg \left(\mathbf{r}-\mathbf{r}^{\prime}\right)$ is the 
angle made by the vector $\mathbf{r}-\mathbf{r}^{\prime}$ and the $x$-direction, (throughout this paper all spatial vectors are projected into the $x y$ plane). A pseudo-particle in a given layer then sees $\phi_{1}$ flux quanta attached to particles in that same layer, and $\phi_{2}$ flux quanta attached to particles in the next layer. In the absence of interlayer tunneling, the relative statistics of particles in different layers is irrelevant, and the statistics of the pseudo-particles depends only on $\phi_{1}$. In particular, the pseudo-particles are bosons if $\phi_{1}$ is odd [11, and fermions if $\phi_{1}$ is even [12].

For the $\nu=1 / 2$ double-layer system there are several interesting choices for $\left(\phi_{1}, \phi_{2}\right)$. For example, if $\left(\phi_{1}, \phi_{2}\right)=(3,1)$ the pseudo-particles obey Bose statistics. When the ChernSimons flux attached to these particles is smeared out according to the standard mean-field prescription [13], each pseudo-boson sees an effective average field $\bar{B}=B_{0}-2 \pi\left(\phi_{1}+\phi_{2}\right) n / e=$ 0 and the applied magnetic field is cancelled exactly. The pseudo-bosons then condense and, just as for the single-layer $\nu=1 / 3 \mathrm{FQHE}$, the resulting condensed state can be shown to be incompressible [11]. The wave function corresponding to this condensed state is, presumably, the so-called 331 wave function, a generalization of Laughlin's wave function for double-layer systems [3 [5]. Exact diagonalization studies have shown that the 331 wave function has a significant overlap with the exact ground state for finite size systems only when $d / l_{0} \lesssim 4$ [4.5]. This is consistent with the fact that the $(3,1)$ scheme becomes untenable in the $d \rightarrow \infty$ limit, because particles in one layer continue to see flux attached to particles in the second layer. To avoid this problem, but retain the mean-field cancellation of the physical field, there is a unique choice: $\left(\phi_{1}, \phi_{2}\right)=(4,0)$. The pseudo-particles are then fermions, and, in the $d \rightarrow \infty$ limit, the layers decouple naturally, so that each layer is described by a compressible 'Fermi liquid' of pseudo-fermions [8,9].

To study the $(4,0)$ mean field theory plus Gaussian fluctuations for finite $d$, it is convenient to formulate the problem in terms of a finite-temperature, Euclidean-time functional integral. For $\left(\phi_{1}, \phi_{2}\right)=(\phi, 0)$, where $\phi=4$ when $\nu=1 / 2$, the Lagrangian density describing the pseudo-fermions is $L(\mathbf{r}, \tau)=L_{0}(\mathbf{r}, \tau)+L_{1}(\mathbf{r}, \tau)$, where the first part of the Lagrangian is 


$$
\begin{aligned}
L_{0}(\mathbf{r}, \tau)=\sum_{s=1,2} & \left(\psi_{s}^{*}(\mathbf{r}, \tau)\left(\partial_{t}-i a_{0}^{(s)}(\mathbf{r}, \tau)\right) \psi_{s}(\mathbf{r}, \tau)\right. \\
& \left.+\frac{1}{2 m_{b}} \psi_{s}^{*}(\mathbf{r}, \tau)\left(-i \boldsymbol{\nabla}+\mathbf{a}^{(s)}(\mathbf{r}, \tau)-e \mathbf{A}_{0}(\mathbf{r})\right)^{2} \psi_{s}(\mathbf{r}, \tau)\right)
\end{aligned}
$$

Here $\psi_{s}$, and $\left(a_{0}^{(s)}, \mathbf{a}^{(s)}\right)$ are, respectively, the pseudo-fermion field and a Chern-Simons gauge field in layer $s, \mathbf{A}_{0}(\mathbf{r})=(\hat{\mathbf{z}} \times \mathbf{r}) B_{0} / 2$ is the physical vector potential describing the applied magnetic field, and $m_{b}$ is the band mass of the electrons. The second part of the Lagrangian,

$$
\begin{aligned}
L_{1}(\mathbf{r}, \tau) & =\sum_{s=1,2} \frac{-i}{2 \pi \phi} a_{0}^{(s)}(\mathbf{r}, \tau) \hat{\mathbf{z}} \cdot\left(\boldsymbol{\nabla} \times \mathbf{a}^{(s)}(\mathbf{r}, \tau)\right) \\
& +\sum_{s, s^{\prime}=1,2} \frac{1}{2(2 \pi \phi)^{2}} \int d^{2} r^{\prime}\left(\boldsymbol{\nabla} \times \mathbf{a}^{(s)}(\mathbf{r}, \tau)\right) V_{s, s^{\prime}}\left(\mathbf{r}-\mathbf{r}^{\prime}\right)\left(\boldsymbol{\nabla} \times \mathbf{a}^{(s)}\left(\mathbf{r}^{\prime}, \tau\right)\right),
\end{aligned}
$$

depends only on the Chern-Simons gauge fields. We work in the Coulomb gauge, $\boldsymbol{\nabla}$. $\mathbf{a}^{(s)}(\mathbf{r}, \tau)=0$, where the first term in (3) is the Chern-Simons term. Integrating out the time components of the Chern-Simons gauge fields then enforces the constraint [1]

$$
2 \pi \phi \psi_{s}^{*}(\mathbf{r}, \tau) \psi_{s}(\mathbf{r}, \tau)=\hat{\mathbf{z}} \cdot\left(\boldsymbol{\nabla} \times \mathbf{a}^{(s)}(\mathbf{r}, \tau)\right)
$$

which describes attaching $\phi$ fictitious $a^{(s)}$ flux quanta to each pseudo-fermion in layer $s$. The second term in (3) is the interlayer and intralayer Coulomb repulsion, where, following Ref. [8], the constraint (4) has been used to rewrite this term purely in terms of the ChernSimons gauge fields. Finally, the Coulomb repulsion itself is

$$
V_{s s^{\prime}}(\mathbf{r})=\frac{2 \pi e^{2}}{\varepsilon \sqrt{r^{2}+d^{2}\left(1-\delta_{s, s^{\prime}}\right)}}
$$

where $\varepsilon$ is the dielectric constant.

At the mean-field level the Chern-Simons gauge fields take their average values, $\left\langle\mathbf{a}^{(s)}(\mathbf{r}, \tau)\right\rangle=2 \pi \phi\left\langle\rho^{(s)}(\mathbf{r})\right\rangle(\hat{\mathbf{z}} \times \mathbf{r})=2 \pi \phi n(\hat{\mathbf{z}} \times \mathbf{r})$, and, for $\nu=1 / 2$ and $\phi=4$, the applied magnetic field is cancelled exactly. The pseudo-fermions in each layer then form Fermi liquids with Fermi wave vector $k_{f}=(4 \pi n)^{1 / 2}=\left(1 / l_{0}\right)(2 / \phi)^{1 / 2}$. Gaussian fluctuations about this mean field state can be studied by integrating out the pseudo-fermion fields in (2), exponentiating the resulting determinant, and expanding to one loop order in the Chern-Simons 
gauge fields, a procedure which is equivalent to the random-phase approximation developed for the anyon gas [13]. The result is an effective action for the Chern-Simons gauge fields,

$$
S_{\mathrm{eff}}[a]=\frac{1}{2} \sum_{n} \int \frac{d^{2} q}{(2 \pi)^{2}} \sum_{\substack{s, s^{\prime}=1,2 \\ \mu, \nu=0,1}} a_{\mu}^{(s)}\left(\mathbf{q}, i \omega_{n}\right) D_{s, \mu ; s^{\prime}, \nu}^{-1}\left(\mathbf{q}, i \omega_{n}\right) a_{\nu}^{\left(s^{\prime}\right)}\left(-\mathbf{q},-i \omega_{n}\right)
$$

where $a_{1}^{(s)}(\mathbf{q}, \omega)=\hat{\mathbf{z}} \cdot\left(\hat{\mathbf{q}} \times\left(\mathbf{a}^{(s)}(\mathbf{q}, \omega)-\left\langle\mathbf{a}^{(s)}(\mathbf{q}, \omega)\right\rangle\right)\right)$ is the fluctuation in the transverse gauge field, and the matrix $D_{s, \mu ; s^{\prime}, \nu}(\mathbf{q}, \omega)$ is the effective propagator for the Chern-Simons gauge fields, the inverse of which is

$$
D_{s, \mu ; s^{\prime}, \nu}^{-1}\left(\mathbf{q}, i \omega_{n}\right)=\left(\begin{array}{cccc}
\Pi_{00}^{0} & \frac{i q}{2 \pi \phi} & 0 & 0 \\
-\frac{i q}{2 \pi \phi} & \Pi_{11}^{0}-\frac{q^{2} V_{11}(q)}{(2 \pi \phi)^{2}} & 0 & -\frac{q^{2} V_{12}(q)}{(2 \pi \phi)^{2}} \\
0 & 0 & \Pi_{00}^{0} & \frac{i q}{2 \pi \phi} \\
0 & -\frac{q^{2} V_{12}(q)}{(2 \pi \phi)^{2}} & -\frac{i q}{2 \pi \phi} & \Pi_{11}^{0}-\frac{q^{2} V_{11}(q)}{(2 \pi \phi)^{2}}
\end{array}\right)
$$

This matrix is labeled according to the scheme $(s, \mu)=[(1,0),(1,1),(2,0),(2,1)]$, where $s$ is the layer index, and $\mu=0,1$ labels the time and transverse component of the ChernSimons gauge fields. Finally, the noninteracting density and transverse-current polarization functions appearing in (77), are, respectively,

$$
\Pi_{00}^{0}=\int \frac{d^{2} k}{(2 \pi)^{2}} \frac{f\left(\epsilon_{\mathbf{k}+\mathbf{q}}\right)-f\left(\epsilon_{\mathbf{k}}\right)}{i \omega_{n}-\epsilon_{\mathbf{k}+\mathbf{q}}+\epsilon_{\mathbf{k}}}
$$

and

$$
\Pi_{11}^{0}=\int \frac{d^{2} k}{(2 \pi)^{2}}\left(\frac{\hat{\mathbf{q}} \times \mathbf{k}}{m_{b}}\right)^{2} \frac{f\left(\epsilon_{\mathbf{k}+\mathbf{q}}\right)-f\left(\epsilon_{\mathbf{k}}\right)}{i \omega_{n}-\epsilon_{\mathbf{k}+\mathbf{q}}+\epsilon_{\mathbf{k}}}-\frac{n}{m_{b}}
$$

where $\epsilon_{\mathbf{k}}=k^{2} / 2 m_{b}-\mu_{f}, \mu_{f}$ is the chemical potential, and $f\left(\epsilon_{\mathbf{k}}\right)$ is the Fermi function.

The collective modes of the system correspond to poles in the gauge field propagator and can be found by solving the equation $\operatorname{det} D^{-1}(q, \omega)=0$. In the limit $\omega \gg k_{f} q / m_{b}$ the analytically continued polarization functions (8) and (9) are $\Pi_{00}^{0} \simeq-\left(n / m_{b}\right)\left(q^{2} / \omega^{2}\right)$ and $\Pi_{11}^{0} \simeq-n / m_{b}$. These expressions can be used to find two propagating modes with dispersion relations 


$$
\omega^{(1)}(q) \simeq \omega_{c}+\frac{e^{2}}{2 \varepsilon \phi} q
$$

and

$$
\omega^{(2)}(q) \simeq \omega_{c}+\frac{e^{2} d}{2 \varepsilon \phi} q^{2}
$$

where $\omega_{c}=2 \pi \phi n / m_{b}$ is the cyclotron frequency. In the limit $q \ll k_{f}, \omega \ll k_{f} q / m_{b}$, the polarization functions are $\Pi_{00}^{0} \simeq m_{b} / 2 \pi$ and $\Pi_{11}^{0} \simeq-\chi_{\mathrm{d}} q^{2}+i k_{f} \omega / 4 \pi q$, where $\chi_{d}=\left(12 \pi m_{b}\right)^{-1}$ is the Landau diamagnetic susceptibility for noninteracting electrons. Again there are two modes, this time diffusive, with dispersion relations

$$
\omega^{(3)}(q) \simeq i \frac{4 e^{2}}{\varepsilon k_{f} \phi^{2}} q^{2}
$$

and

$$
\omega^{(4)}(q) \simeq i \frac{4 \pi \tilde{\chi}}{k_{f}} q^{3}
$$

where

$$
\tilde{\chi}=\frac{\left(1+e^{2} m_{b} d\right)}{2 \pi \phi^{2} m_{b}}+\frac{1}{12 \pi m_{b}} .
$$

It is interesting to compare these modes with those which appear in the Fermi-liquid description of the $\nu=1 / 2$ single-layer system [8]. When the long-range Coulomb repulsion between electrons is properly taken into account, the collective modes of the single-layer system have the same dispersions as modes 1 and 3, while for short-ranged interactions they have the same dispersions as modes 2 and 4 [8]. This correspondence is not surprising, because in the double-layer system modes 1 and 3 involve density fluctuations which are in phase in the two layers, while modes 2 and 4 involve density fluctuations which are out of phase, and hence are unaffected by the long-range nature of the Coulomb repulsion. We note in passing that the high-energy propagating modes 1 and 2 are the in-phase, and outof-phase magneto-plasmons, or Kohn's modes, and (10) is consistent with Kohn's theorem [14, which requires that the energy of mode 1 go to the unrenormalized cyclotron frequency, 
$\omega_{c}$, as $q \rightarrow 0$. Within the random-phase approximation, the energy of mode 2 also goes to $\omega_{c}$ as $q \rightarrow 0$, and while this is not required by Kohn's theorem, which follows from translational symmetry and thus only applies to the in-phase mode, it does reflect the 'decoupled' nature of the $(4,0)$ state.

As in the single-layer system, the diffusive modes 3 and 4 are the most important source of low-energy quasiparticle scattering. To study these modes it is useful to define the symmetric and antisymmetric transverse Chern-Simons gauge fields: $a_{1}^{( \pm)}=\left(a_{1}^{(1)} \pm a_{1}^{(2)}\right) / \sqrt{2}$. The retarded propagators for these fields are found by performing the matrix inversion (7) and analytically continuing to the real frequency axis. In the $q \ll k_{f}$, $\omega \ll k_{f} q / m_{b}$ limit, the resulting propagators are

$$
D_{11 ;+}(\mathbf{q}, \omega) \simeq \frac{1}{2}\left[\frac{e^{2}}{\pi \phi^{2}} q-i \frac{k_{f} \omega}{4 \pi q}\right]^{-1}
$$

and

$$
D_{11 ;-}(\mathbf{q}, \omega) \simeq \frac{1}{2}\left[\tilde{\chi} q^{2}-i \frac{k_{f} \omega}{4 \pi q}\right]^{-1}
$$

for the symmetric and antisymmetric transverse Chern-Simons gauge fields, respectively. At low frequencies and long wavelengths, the effective interaction between pseudo-fermions is dominated by $D_{11 ;-}$, (all other components of $D$ are less singular for small $q$ ), and has the form

$$
V_{s s^{\prime}}^{\mathrm{eff}}\left(\mathbf{k}, \mathbf{k}^{\prime} ; \mathbf{q}, \omega\right) \simeq\left(\begin{array}{rr}
-1 & 1 \\
1 & -1
\end{array}\right) \frac{(\mathbf{k} \times \hat{\mathbf{q}}) \cdot\left(\mathbf{k}^{\prime} \times \hat{\mathbf{q}}\right)}{m_{b}^{2}} \frac{1}{2}\left[\tilde{\chi} q^{2}-i \frac{k_{f} \omega}{4 \pi q}\right]^{-1}
$$

where the matrix is a layer matrix. It follows from (14) that this interaction grows stronger with decreasing $d$. Precisely such a singular current-current interaction appears in the $\nu=$ $1 / 2$ single-layer system for the physically unrealistic case of short-ranged electron-electron interactions [8]. In the double-layer system, not only does this interaction appear even when the long-range Coulomb repulsion is included, but, when $\mathbf{k}^{\prime}=-\mathbf{k}$, i.e., in the Cooper channel, the effective interaction between pseudo-fermions in different layers is attractive. 
Physically, this attractive pairing interaction appears because $a_{1}^{(-)}$couples to pseudofermions in different layers as if they were oppositely charged. Thus, while the coherent propagation of a single pseudo-fermion is strongly inhibited by the random, path dependent Aharonov-Bohm phase coming from fluctuations in $a_{1}^{(-)}$, a pair of pseudo-fermions, one from each layer, can propagate coherently through these fluctuations, because the AharonovBohm phase seen by one pseudo-fermion exactly cancels that seen by the second. The physics here is remarkably similar to the problem of holes constrained to hop on the same sublattice of a quantum disordered antiferromagnet; a problem which can be mapped onto an effective field theory in which holes on different sublattices interact via a fictitious gauge field as if they were oppositely charged [15]. In this problem, a pair condensate is expected to appear [16], and it is interesting to note that if a similar pseudo-fermion pair condensate, $\left\langle\psi_{1}^{*} \psi_{2}^{*}\right\rangle \neq 0$, were to appear in the $\nu=1 / 2$ double-layer system, it would correspond to a FQHE for the original electrons, in the same way that the $\nu=1 / 3$ single-layer FQHE can be understood in terms of the condensation of pseudo-bosons [11. Such a pairing scenario is similar to that considered by Greiter et al. [17] for the spin-polarized $\nu=1 / 2$ state, where pairing of like spin electrons in the $p$-wave channel was argued to lead to a single-layer spin-polarized $\nu=1 / 2 \mathrm{FQHE}$. However, in the double-layer system it is unlikely that the transition from the $(4,0)$ 'metallic' phase to the $(3,1)$ 'condensed' phase can be understood simply in terms of the effective pairing interaction (17), particularly because this interaction changes when the system becomes superconducting. In fact, the experimental evidence for a compressible phase when the layer spacing is large enough indicates that, in general, this pairing instability does not occur. Nevertheless, the above arguments suggest that there should be pairing fluctuations present in the compressible phase of a $\nu=1 / 2$ double-layer system; fluctuations which grow stronger with decreasing $d$, and which, perhaps, play some role in the eventual instability of the $(4,0)$ phase to the $(3,1)$ phase.

A rough measure of the relative importance of fluctuations in $a_{1}^{(+)}$and $a_{1}^{(-)}$can be found by calculating the scattering rates due to these two types of fluctuations for a pseudo- 
fermion with initial energy $\epsilon_{\mathbf{k}}$. Following similar analyses in the literature [8, 18, we use Fermi's golden rule to obtain

$$
\frac{1}{\tau_{k}^{+}} \simeq \frac{k_{f} \varepsilon \phi^{2}}{8 m_{b} e^{2}} \epsilon_{\mathbf{k}}
$$

for scattering from the symmetric fluctuations, and

$$
\frac{1}{\tau_{k}^{-}} \simeq \frac{3 \sqrt{3}}{32 \pi}\left(\frac{8 \pi E_{f}}{m_{b}^{2} \chi^{2}}\right)^{1 / 3} \epsilon_{\mathbf{k}}^{2 / 3}
$$

for scattering from the antisymmetric fluctuations. Here $E_{f}=k_{f}^{2} / 2 m_{b}=\omega_{c} / \phi$ is the meanfield Fermi energy. Because $a_{1}^{(-)}$leads to more singular scattering than $a_{1}^{(+)}$, there is a region around the Fermi surface, $\left|k^{2} / 2 m_{b}-\mu_{f}\right| \lesssim E_{c}$, for which scattering from $a_{1}^{(-)}$is dominant, where

$$
\frac{E_{c}}{E_{f}} \simeq \frac{(3 \sqrt{3})^{3}}{4} \frac{1}{\phi^{2}\left(1+\phi^{2} / 6+d / a_{0}^{*}\right)^{2}\left(k_{f} a_{0}^{*}\right)^{3}}
$$

Here all dependence on the band mass has been absorbed into $a_{0}^{*} \equiv \varepsilon / e^{2} m_{b}$, the effective Bohr radius, $\left(a_{0}^{*} \simeq 82 \AA\right.$ for GaAs). As noted in Ref. [8], in the extreme quantum limit, where $a_{0}^{*} \gg l_{0}$, the kinetic energy is completely quenched by the applied field and the band mass should not appear in any physically relevant low-energy quantities. In this limit we expect that corrections beyond the random-phase approximation will effectively renormalize $a_{0}^{*}$ to $l_{0}$ in (20). However, for the experiments discussed in [1], $l_{0} \sim a_{0}^{*}$, and the system is not in the extreme quantum limit. Accordingly in what follows we have used (20) without modification. Table I summarizes the parameters $k_{f} a_{0}^{*}, d / a_{0}^{*}, E_{f}$ and $E_{c} / E_{f}$ which characterize the four samples discussed in Ref. [1]. For samples A, B, and C, which exhibit the FQHE, and which are, presumably, in the $(3,1)$ phase, $E_{c}$ becomes smaller as the observed FQHE weakens. And for sample D, which does not show the FQHE at all, $E_{c}$ has the smallest value of all four samples. This is consistent with the hypothesis that the out-of-phase fluctuations are related to the instability of the $(4,0)$ phase to the $(3,1)$ phase.

To conclude, the compressible phase of a double-layer electron system in a transverse magnetic field with total Landau-level filling factor $\nu=1 / 2$ has been studied. Following 
Halperin, Lee, and Read [8], and Kalmeyer and Zhang [9], the system was transformed into a mathematically equivalent system of pseudo-fermions in zero average magnetic field interacting via a Chern-Simons gauge field, as well as the interlayer and intralayer Coulomb repulsion. The two layers decouple naturally in the $d \rightarrow \infty$ limit, but for finite $d$ the interlayer Coulomb repulsion gives rise to a new, low-lying diffusive mode. This new mode leads both to more singular low momentum scattering than occurs in the single layer case, and to an attractive pairing interaction between pseudo-fermions in different layers. The appearance of this attractive interaction, which grows stronger with decreasing $d$, may be related to the experimentally observed instability of the compressible $(4,0)$ phase to an incompressible FQHE state.

I would like to acknowledge useful discussions with D. Khveshchenko, D.H. Lee, L. Lilly, M. Reizer, J.R. Schrieffer, F.C. Zhang, and S.C. Zhang. This work was supported by NSF Grant No. DMR-91-14553 and by the National High Magnetic Field Laboratory at Florida State University. 


\section{TABLES}

TABLE I. Sample parameters from Ref. [1]. $a_{0}^{*}$ is the effective Bohr radius, $d$ is the layer spacing, $k_{f}$ and $E_{f}$ are the mean-field Fermi wave vector and energy, and $E_{c}$ is the energy scale defined in (20).

\begin{tabular}{ccccccc}
\hline \hline Sample & $d / a_{0}^{*}$ & $k_{f} a_{0}^{*}$ & $\begin{array}{c}E_{f} \\
(\mathrm{meV})\end{array}$ & $\begin{array}{c}E_{c} \\
(\mathrm{meV})\end{array}$ & $E_{c} / E_{f}$ & $\begin{array}{r}\text { Strength } \\
\text { of } \nu=1 / 2\end{array}$ \\
\hline $\mathrm{A}$ & 2.6 & 0.66 & 3.4 & 0.67 & 0.19 & Strongest \\
$\mathrm{B}$ & 2.6 & 0.75 & 4.5 & 0.60 & 0.13 & Strong \\
$\mathrm{C}$ & 2.6 & 0.80 & 5.1 & 0.56 & 0.11 & Weak \\
$\mathrm{D}$ & 3.4 & 0.75 & 4.5 & 0.47 & 0.10 & Absent \\
\hline \hline
\end{tabular}




\section{REFERENCES}

[1] J.P. Eisenstein, G.S. Boebinger, L.N. Pfeiffer, K.W. West and Song He, Phys. Rev. Lett., 68, 1383 (1992).

[2] Y.W. Suen, L.W. Engel, M.B. Santos, M. Shayegan, and D.C. Tsui, Phys. Rev. Lett., 68, $1379(1992)$

[3] B.I. Halperin, Helv. Phys. Acta 56,75 (1983)

[4] D. Yoshioka, A.H. McDonald, and S.M. Girvin, Phys. Rev. B, 39, 1932 (1989).

[5] S. He, X.C. Xie, S. Das Sarma, and F.C. Zhang, Phys. Rev. B, 43, 9339 (1991).

[6] F.D.M. Haldane and E.H. Rezayi, Phys. Rev. Lett. 60, 956 (1988).

[7] H.W. Jiang, H.L. Stormer, D.C. Tsui, L.N. Pfeiffer, and K.W. West, Phys. Rev. B 40, 12013 (1989); R.L. Willett, M.A. Paalanen, R.R. Ruel, K.W. West, L.N. Pfeiffer, and D.J. Bishop, Phys. Rev. Lett. 65, 112 (1990); T. Sajoto, Y.W. Suen, L.W. Engel, M.B. Santos, and M. Shayegan, Phys. Rev. B 41, 8449 (1990).

[8] B.I. Halperin, P.A. Lee, and N. Read, Phys. Rev. B, 47, 7312 (1993).

[9] V. Kalmeyer and S.C. Zhang, Phys. Rev. B, 46, 9889 (1992).

[10] F. Wilczek, Phys. Lett. 48, 1144 (1982).

[11] S.C. Zhang, T.H. Hansson, and S. Kivelson, Phys. Rev. Lett. 62, 82 (1989); D.H. Lee and S.C. Zhang, ibid. 66, 1220 (1991); S.C. Zhang, Int. J. Mod. Phys. B 6, 25 (1992).

[12] J.K. Jain, Phys. Rev. Lett. 63, 199 (1989); Phys. Rev. B 40, 8079 (1989); 41, 7653 (1990).

[13] A.L. Fetter, C. B. Hanna, and R. B. Laughlin, Int. J. Mod. Phys. B 5, 2751 (1991); Y. H. Chen, B.I. Halperin, F. Wilczek, and E. Witten, Int. J. Mod. Phys. B 3, 1001 (1989). 
[14] W. Kohn, Phys. Rev. 123, 1242 (1961).

[15] P.A. Lee, Phys. Rev. Lett. 63, 680 (1989); X.G. Wen, Phys. Rev. B 39, 7223 (1989).

[16] B. Normand, P.A. Lee, and N. Nagaosa, Physica C 185-189, 1479 (1991); D.V. Khveshchenko, preprint.

[17] M. Greiter, X.G. Wen, and F. Wilczek, Phys. Rev. Lett. 66, 3205 (1991); Nucl. Phys. B, 374, 567 (1992).

[18] P.A. Lee and N. Nagaosa, Phys. Rev. B 46, 5621 (1992). 